\title{
Article
}

\section{A Paradox of Unity}

\section{Jens V. Fischer}

German Aerospace Center (DLR), Microwaves and Radar Institute, 82234 Wessling, Germany; jens.fischer@dlr.de, Tel.: +49-8153-28-3057

\begin{abstract}
In previous studies we found that generalized functions can be smooth, discrete, periodic or discrete periodic and they can either be local or global and they are regular or generalized functions. We also saw that these properties were related to Poisson's summation formula on one hand and to Heisenberg's uncertainty principle on the other. In this paper, we interlink these studies and show that scalars (real or complex numbers) considered as trivial functions are discrete and periodic, local and global as well as regular and generalized, simultaneously. However, this is also a paradox because it means that Dirac's $\delta$ and 1 (its Fourier transform) coincide. They both are unity. We show that $\delta$ and 1 coincide in the sense of scalars (real or complex numbers) but they differ in the sense of (generalized) functions. This result can moreover be related to Max Born's principle of reciprocity. It also answers an open question in present-day quantum mechanics because it means that the Dirac delta squared is simply delta.
\end{abstract}

Keywords: Dirac impulse; generalized functions; tempered distributions; paradox of unity; Dirac's delta squared; quantum mechanics; Born's principle of reciprocity; scalar self-reciprocity

MSC: 42B05, 42B08, 42B10, 46F05, 46F10

\section{Introduction}

It is commonly known today that the Fourier transform of a Dirac comb is a Dirac comb [1-8]. This alone is very interesting. Because if we think of a Dirac comb as a sampled constant function 1 then its Fourier transform is again a sampled constant function 1 . Symbolically, we may write $\mathcal{F}(\Perp 1)=\Perp 1$ where $\amalg$ denotes sampling at integers and $\amalg 1$ denotes sampling at integers applied to 1 which is a Dirac comb [9]. In other words, the Fourier transform of a "discrete" 1 is a "discrete" 1.

Transferring this finding to a "smooth" 1 , i.e., to the ordinary real number 1 , it means that the Fourier transform of 1 should be 1 and, indeed, this result is obtained if it turned out that Dirac's $\delta$ and 1 are the same number. We already know that the Fourier transform of 1 is a Dirac delta, symbolically

$$
\mathcal{F} 1=\delta
$$

and

$$
\mathcal{F} \delta=1
$$

where $\mathcal{F}$ is defined according to the "unitary, ordinary frequency" Fourier transform convention [10].

We start from $\delta$, trivially being multiplied by a constant function 1 , which according to the sampling property [11] of $\delta$, given in (11), (12), is

$$
\delta=\delta \cdot 1=1 .
$$

In other words, sampling 1 at the origin yields 1 . One may think of this formula as depicted in Figure 1 , Figure 3 or Figure 4. It is moreover true in the discrete case. An equivalent "fully discrete" formulation of (1) is

$$
1=[1,0, \ldots, 0]^{T} \cdot[1,1, \ldots, 1]=1,
$$

where $[1,0, \ldots, 0]^{T}$ is a column vector representing the Dirac impulse at zero position, $[1,1, \ldots, 1]$ denotes the discrete constant function 1 , and · represents the dot product, also known as inner product 
or scalar product in $\mathbb{R}^{n}$. By "fully discrete" we mean that $\delta$ and 1 are discrete in both, time and frequency domain (Figure 2). It results in n-tuples (vectors) as explained the next section.

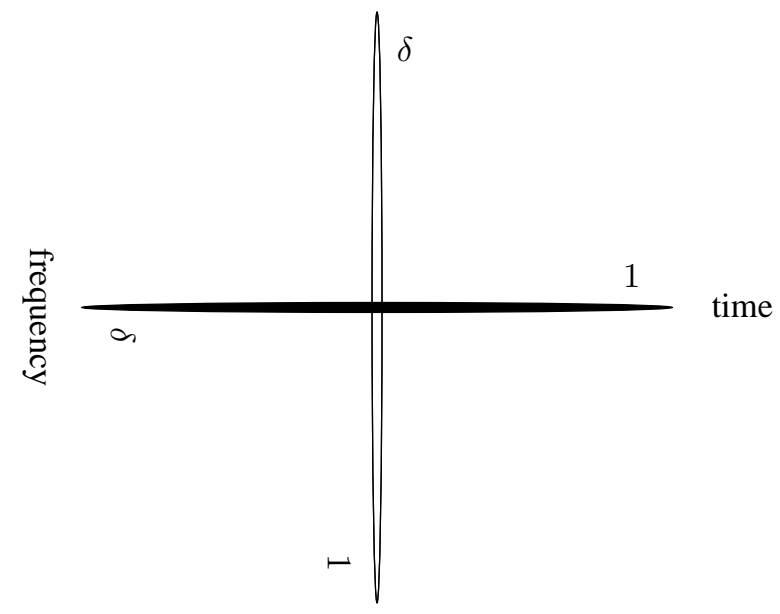

Figure 1. A unit mass as observed in time and frequency domain. Its shape in frequency domain depends on whether we think of it as focused (white) or defocused (black) entity. In contrast to that it remains both, focused and defocused simultaneously (scalar), as long as we do not decide its shape.

Back to the "fully smooth" case, let us Fourier transform (1). It yields

$$
\mathcal{F}(\delta)=\mathcal{F}(\delta \cdot 1)=\mathcal{F}(\delta) * \mathcal{F}(1)=1 * \delta=1
$$

where $\delta$ is the neutral element with respect to convolution. But, more generally, the latter equality also holds on constants. Let $c$ be any complex number then it can of course be written

$$
c * \delta=c
$$

or, using the Fourier transform, alternatively as

$$
c \cdot 1=c
$$

where we think of $c$ as a constant function. Moreover, in contrast to that and according to the definition of convolution, we also have

$$
c * 1=c
$$

where we think of $c$ as an ordinary number that has been taken as the "weights" for a function that is constantly 1. Fourier transforming this formula yields

$$
c \cdot \delta=c
$$

which is also true because sampling a constant $c$, wheresoever, yields a scalar $c$. One may observe that (3) is more general than (1). This approach of testing generalized functions such as $\delta$ or 1 on constant "test functions" will be needed later. It follows the central idea of distribution theory [12-17] where any "action" of generalized functions is tested on smooth functions.

In Section 2, we first summarize our results so far and provide a brief introduction to the theory of generalized functions in Appendix 7 and to definitions used in Appendix 8. Section 3 then presents the two central theorems in this study and Section 4 as well as Appendix 9 relate these results to quantum mechanics and relativity theory. In Section 5, we describe a few applications. Conclusions are finally given in Section 6. 


\section{Preliminaries}

In a first study, we introduced discretization $\Perp$ and periodization $M$ within the space of tempered distributions $\mathcal{S}^{\prime}$ and showed that periodizing a function in "time domain" means discretizing a function in "frequency domain". In that way, functions can be converted from being fully smooth (i.e. smooth in both, time and frequency domain) to functions which are being fully discrete (i.e. discrete in both, time and frequency domain), i.e., to vectors, matricies or tensors (depending on whether they depend on one, two or more variables) by applying discretization and periodization, respectively [18].

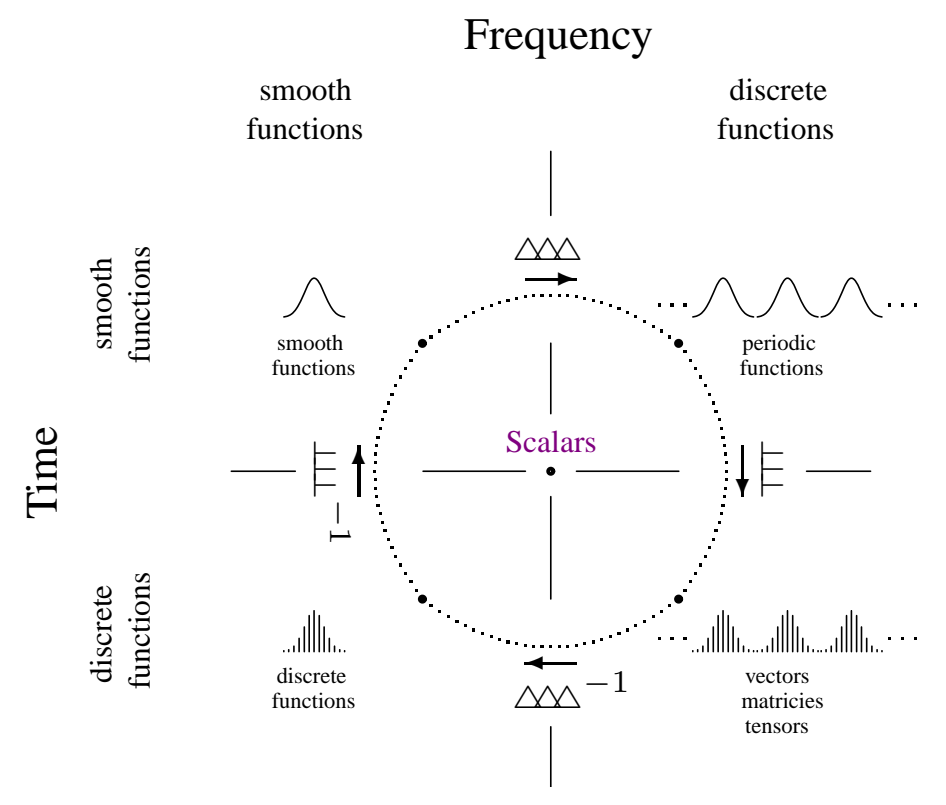

Figure 2. Scalars are discrete and smooth in both, time and frequency domain.

Vice versa, fully discrete functions (vectors, matricies or tensors) can be converted back to fully smooth functions via inverse periodization (localization) and inverse discretization (regularization) [19]. It followed that the space of tempered distributions falls into four parts (or three if the two mixed spaces are combined to a space of "half-discrete" functions) [20]:

$$
\mathcal{S}^{\prime}=\mathcal{S}_{S S}^{\prime} \cup\left(\mathcal{S}_{S D}^{\prime} \cup \mathcal{S}_{D S}^{\prime}\right) \cup \mathcal{S}_{D D}^{\prime}
$$

$\mathcal{S}_{S S}^{\prime}$ includes functions which are smooth in both time and frequency domain (it coincides with $\mathcal{S}$, the Schwartz space), $\mathcal{S}_{S D}^{\prime}$ includes functions which are smooth in time and discrete in frequency domain, $\mathcal{S}_{D S}^{\prime}$ includes functions which are discrete in time and smooth in frequency domain and $\mathcal{S}_{D D}^{\prime}$ includes functions which are discrete in both time and frequency domain. We will now see that - as an exception - scalars belong to all these spaces (Figure 2).

\section{A Paradox of Unity}

One may already have noticed that (1) looks like a contradiction. It cannot be explained, at least, with everything we know so far. We now prove this formula using rigorous distributional means. Readers unfamiliar with the applied proof technique may refer to Appendix 7. 


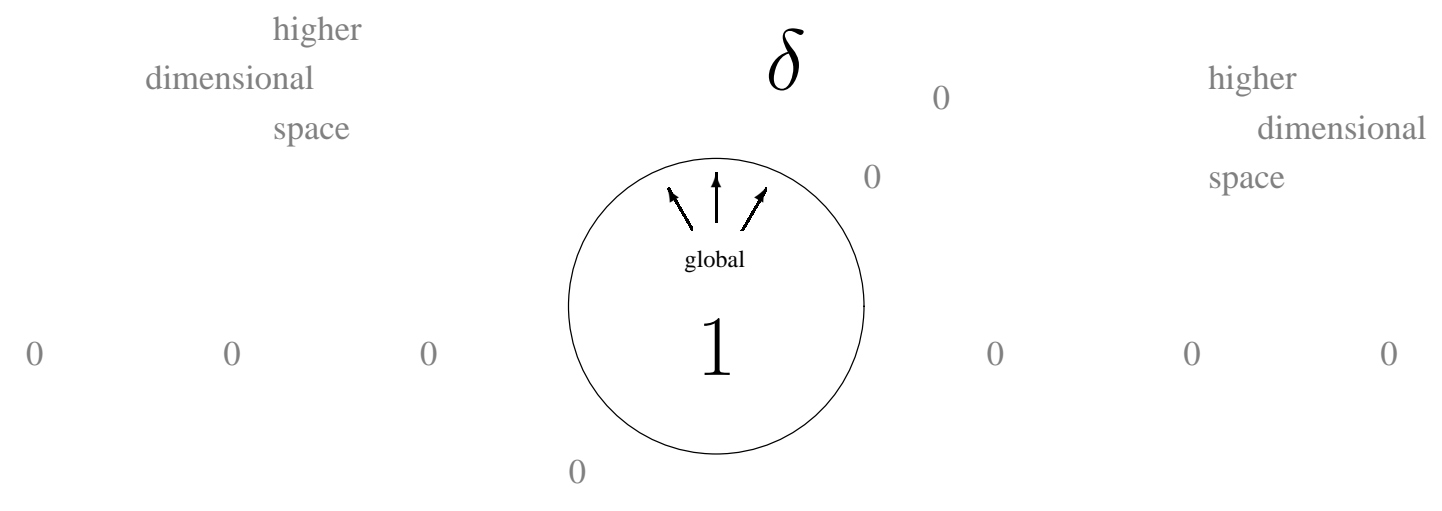

Figure 3. A unit mass. It is trivially embedded in all higher dimensional spaces. Outside unity it is $\delta$, inside unity it is 1 . From outside it is local, from inside it is global and the Fourier transform switches from inside to outside $\mathcal{F} 1=\delta$ and from outside to inside $\mathcal{F} \delta=1$. This way of thinking remains true in arbitrary dimensional, either smooth or discrete spaces, even in zero-dimensional spaces.

Theorem 1. Let $\delta$ be Dirac's delta and 1 its Fourier transform, then

$$
\delta=1
$$

in the sense of scalars (real or complex numbers).

Proof. Applying $\delta \in \mathcal{E}^{\prime}$ to any constant test function $c \in \mathcal{E}$ we obtain

$$
\langle\delta, c\rangle=c(0)=c=\langle 1, c\rangle
$$

i.e., $\delta$ and 1 coincide in the sense of constant functions.

Theorem 2. Let $\delta$ be Dirac's delta and 1 its Fourier transform, then

$$
\delta \neq 1
$$

in the sense of (generalized) functions.

Proof. Applying $\delta \in \mathcal{E}^{\prime}$ to any non-constant test function $\varphi \in \mathcal{E}$ we obtain

$$
\langle\delta, \varphi\rangle=\varphi(0) \neq \varphi=\langle 1, \varphi\rangle
$$

i.e., $\delta$ and 1 differ in the sense of generalized functions.

Theorem 1 tells us that whenever unity is meant as scalar, its Fourier transform is again scalar. But if unity is meant as function then it is $\delta$ or the function that is constantly 1 . Hence, their Fourier transforms are 1 or $\delta$, respectively. Although this might seem paradoxical at first sight, Theorem 1 and Theorem 2 do not contradict each other. They even complement each other. This can be seen by 
Table 1. Fourier Transforms of Unity

\begin{tabular}{|c|c|c|}
\hline No & Rule & Remark \\
\hline 1 & $\mathcal{F}\left(\Perp_{\frac{1}{T}} f\right)=T{\aleph_{T}}_{T}(\mathcal{F} f)$ & Poisson Summation Formula \\
\hline 3 & $\mathcal{F}(\amalg f)=\bowtie(\mathcal{F} f)$ & abbreviated for $T=1$ \\
\hline 5 & $\mathcal{F}(\Perp 1)=\triangle \triangle \delta$ & where $\mathcal{F} 1=\delta$ \\
\hline 7 & $\mathcal{F}(\Perp 1)=\Perp 1$ & $\triangle \delta=\Perp 1$ \\
\hline 9 & $\mathcal{F} 1=\delta$ & 1 is smooth \\
\hline 11 & $\mathcal{F} 1=1$ & 1 is scalar \\
\hline 10 & $\mathcal{F} \delta=1$ & 1 is smooth \\
\hline 8 & $\mathcal{F}(\triangle \Delta \delta)=\triangle \Delta \delta$ & $\Perp 1=\triangle \Delta \delta$ \\
\hline 6 & $\mathcal{F}(\triangle \Delta \delta)=\Perp 1$ & where $\mathcal{F} \delta=1$ \\
\hline 4 & $\mathcal{F}(\bowtie f)=\Perp(\mathcal{F} f)$ & abbreviated for $T=1$ \\
\hline 2 & $\mathcal{F}\left({\aleph_{T}}_{T} f\right)=\frac{1}{T} \uplus_{1}(\mathcal{F} f)$ & Poisson Summation Formula \\
\hline
\end{tabular}

looking at their proofs. We either used constant or non-constant functions taken from the test function space $\mathcal{E}$ of $\delta \in \mathcal{E}^{\prime}$ in order to check its impact on functions.

The following corollary is an immediate consequence of Theorem 1 .

Corollary 1. Let $\mathcal{F}$ be the "unitary, ordinary frequency" Fourier transform (in the distributional sense) and let 1 be unity (real number). Then

$$
\mathcal{F} 1=1
$$

in the sense of scalars.

It follows immediately from rules $\mathcal{F} \delta=1$ and $\mathcal{F} 1=\delta$ which are commonly known. Table 1 summarizes all rules found so far. Generalizing this, we also have the following statement.

Corollary 2. Let $\mathcal{F}$ be the "unitary, ordinary frequency" Fourier transform (in the distributional sense) and let $c$ be any complex number. Then

$$
\mathcal{F}_{C}=c
$$

in the sense of scalars.

The latter is implied by the linearity of $\langle\cdot, \cdot\rangle$ and of ordinary integrals. Property (7) is also expressed by saying: Scalars are self-reciprocal, as we will see later. Let us now try to better understand these results. We may interpret them in the following way.

Unity arises in three different ways. It can be scalar, focused or defocused (Figure 1). Alternatively, we may say it can be scalar, local or global (Figure 3) and, equivalently, we may say it can be scalar, discrete or smooth (Figure 4). Additionally, we may say it arises as scalar (ordinary number), as regular function (constantly one) or as generalized function (Dirac delta). Within its own, zero-dimensional space it is even scalar and discrete (focused, local) and smooth (defocused, global), simultaneously. In contrast to that, in any higher-dimensional space, the properties of being "local" and "global" are virtually ripped apart due to the extent of the space and then swapped by the Fourier transform (Figure 4). We may therefore think of $\delta$ as the space's inner "kernel" (most local element) and of 1 as its outer "sky" (most global element) or a "ball" representing the whole space (Figure 5). Discreteness arises as soon as we think of unity as if it where "taken out of its context" (embedded in zeros) and smoothness arises as soon as we think of unity as if it where "repeated in all dimensions" (padded by itself). Recall that we already encountered a discreteness-periodicity duality in [18] and a locality-globality duality 
in [19]. They are expressed in Poisson's summation formula and in Heisenberg's uncertainty principle, respectively.

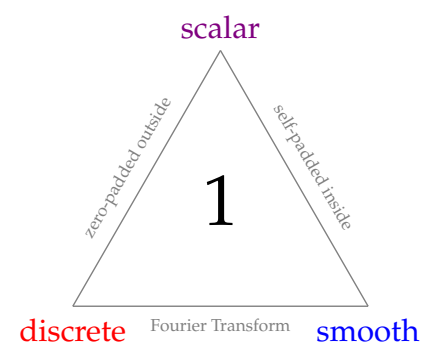

Figure 4. Three shapes of unity.

Replacing "discrete" by "generalized function" and "smooth" by "regular function" in Figure 4, we see that it actually yields formula (1) and therewith reflects the overall idea of distribution theory where generalized functions applied to smooth functions yield scalars, see Appendix 7, eq. (14). Hence, all distribution theory melts down at unity by applying $\delta$ to constantly 1 yielding scalar 1 , expressed in Figure 4, (1), (2), (8) and (14).

\section{The Principle of Reciprocity}

It has already been suggested in Figure 3 to think of the Fourier transform as an operation that allows switching from a scalar's inside to its outside and, vice versa, from its outside to its inside. More generally, it even seems that higher-dimensional spaces behave like individual scalars (Figure 5).

One may observe furthermore that any positively expanding space (Figure 5, right) is mirrored by a negatively expanding space (Figure 5 , left) where inside and outside are swapped. This can be thought of letting a clock run forward in one space ("time" running forwards), and to simultaneously look at the same clock from the other side: It runs backwards, i.e. "frequency" runs forward in this space. Scalars are obviously fixpoints of the Fourier transform (Figure 5, middle) because they are not structured at all, i.e., inside equals outside. The following notation will therefore be advantageous (one may compare this to Figure 4):

$$
1 \in \mathbb{R}^{-n} \text { which is a Dirac } \delta \text { applied to constantly one } 1 \in \mathbb{R}^{+n} \text { yields } 1 \in \mathbb{R}^{0},
$$

i.e., the space dimensions $-n$ and $+n$ add up to 0 . Negative-dimensional spaces are motivated in section 5.1, multi-dimensional scalars (e.g. $1 \in \mathbb{R}^{n}$ ) are proposed in 5.2 and an example for $\delta$ in $\mathbb{R}^{2}$ discretized with $n=4$ in both dimensions is explicitely given in Section 5.3.

Looking at Figure 5, the reader may feel reminded of "big bang theory" and the history of our universe. One may also see mother Earth from "inside", looking at its blue sky and being aware of its hot inner core (Figure 5, right). Simultaneously, one may see mother Earth as blue dot from "outside" and being aware of the homogeneously distributed, still "warm" cosmic background radiation (Figure 5, left). There is indeed a striking parallel to all these things which is expressed in Max Born's principle of reciprocity [21-26]. 


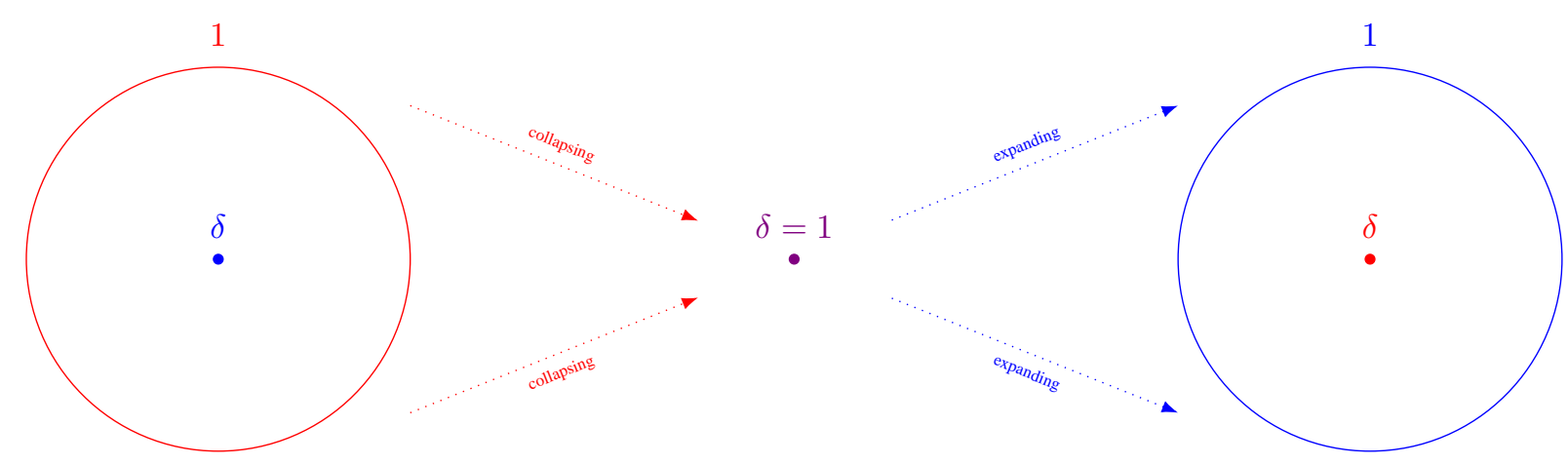

Figure 5. Unity is scalar (middle) in zero-dimansional spaces. But in higher-dimensional spaces (left and right), it either appears locally as "dot" $\delta$ seen from outside (left) or globally as "sky" 1 seen from inside (right). The Fourier transform, moreover, allows switching between inner and outer spaces (left: "frequency domain" and right: "time domain"), i.e., from $\delta$ to 1 and from 1 to $\delta$. Additionally, one may find beside $\delta$ an omnipresent "background" 1 (left) or beside 1 a "space kernel" $\delta$ (right).

He proposed this principle in the endeavour to unify laws applicable in microscopic (quantum theory) and macroscopic dimensions (relativity). Einstein's famous formula $E=m c^{2}$, for example, appears a bit unbalanced. One may, however, rewrite it as

$$
E c^{-1}=m c^{+1}
$$

to better emphasize its "reciprocity". We may think of $c^{-1}$ as "time" (seconds per unit) and of $c$ as "frequency" (units per second). Einstein's formula now reminds us of the energy conservation law between "time" and "frequency" domain, Parseval's theorem [27]. Another thought that comes into mind is that the "difference" between $c^{-1}$ and $c^{+1}$ actually measures (twice) the "distance" from "big-bang" to where we are (Figure 5), in other words, the "time" that has past since then. Thinking about the paradox of unity, it would moreover be interesting to go back to where "time was zero" by letting the velocity of light be unity, hence $c=1$, and despite this seems absurd, it is ideed ocassionally done, for example in $[28,29]$, to achieve symmetry between momentum-energy and space-time.

Max Born proposed amongst others also splitting up Planck's constant [28]

$$
\hbar=a b
$$

into two factors $a$ and $b$ each absorbing half of all units such that momentum-energy and space-time appear in true balance and unitless. "There is strong formal evidence for the hypothesis, which I have called the principle of reciprocity, that the laws of nature are symmetrical with regard to space-time and momentum-energy ..." He moreover wrote "The property of self-reciprocity can also be expressed by saying that [a function] is its own Fourier transform." and that "These eigenfunctions [of the Fourier transform] represent all possible self-reciprocal scalars, vectors, and tensors." [28]. In other words, he postulated that even scalars can be self-reciprocal - as found in Corollary 1 and Corollary 2.

\section{Applications}

\subsection{Negative Dimensional Spaces}

The considerations above propose an introduction of zero-dimensional and negative-dimensional spaces. We introduce $\mathbb{R}^{0}$ and $\mathbb{C}^{0}$ as the spaces of real-valued and complex-valued scalars, respectively, and define negative-dimensional spaces as the spaces whose Fourier transform is positive-dimensional. This definition is moreover justified by the fact that in positive-dimensional spaces, time $t^{+1}$ is running 
forwards (towards infinity) while in negative-dimensional spaces, reciprocal time $t^{-1}$ is running backwards (towards zero). The latter can also be expressed by saying that "frequency" is running towards infinity. However, there is no possibility whatsoever to absolutly determine which of the two spaces should be called positive or negative dimensional. Their orientability rather behaves like a Möbius band [20]. A minus sign therefore simply expresses that it is the Fourier transform of another space or, equivalently, that "time" is running in an opposite direction. The direction of "time" is also discussed at the end of Appendix 9.

\subsection{Multi-dimensional Scalars}

It has already been found that scalars may arise in different forms [30]. They can be multi-dimensional, for example. A convention of negative-dimensional spaces as mentioned above excellently allows now distinguishing scalar, local and global unities. We may say that $1 \in \mathbb{R}^{0}$ defines a scalar unity, $1 \in \mathbb{R}^{n}$ defines a global unity (e.g. n-tuple $[1,1, \ldots, 1]$ ) and $1 \in \mathbb{R}^{-n}$ defines a local unity (e.g. Dirac delta $[1,0, \ldots, 0]$ in $\mathbb{R}^{n}$ ). One may say that, unless otherwise indicated, a minus sign refers to the Dirac delta within a given space. In that way we are ready to distinguish two kinds of multi-dimensional scalars beside the usual comprehension of zero-dimensional scalars.

\subsection{Zero-Padding}

The usual technique to produce multi-dimensional scalars from zero-dimensional ones is called "zero-padding". It is wide-spread in digital signal and digital image processing because it allows interpolating, i.e., to zoom into data. Zero-padding in one (time or frequency) domain is a trivial form of discretization. It yields interpolation in the other domain [19]. Applied to a scalar, this interpolation becomes periodization.

Zero-padding a scalar 1 in "time" domain along $\mathbb{R}^{1}$ yields for example (we discretize at integers and periodize after $n=4$ samples)

$$
[1,0,0,0]
$$

or along $\mathbb{R}^{2}$ it yields (we discretize at integers and periodize after $n=4$ samples)

$$
\left[\begin{array}{llll}
1 & 0 & 0 & 0 \\
0 & 0 & 0 & 0 \\
0 & 0 & 0 & 0 \\
0 & 0 & 0 & 0
\end{array}\right]
$$

whereas the "frequency" domain (it was the scalar 1 initially) becomes

$$
[1,1,1,1]
$$

and

$$
\left[\begin{array}{llll}
1 & 1 & 1 & 1 \\
1 & 1 & 1 & 1 \\
1 & 1 & 1 & 1 \\
1 & 1 & 1 & 1
\end{array}\right]
$$

respectively. More correctly, the latter two structures are additionally divided by the number of their elements (4 and 16, here) to fulfill Parseval's theorem [27]. One may furthermore observe that these structures are being cyclic, i.e., their left and their right margin as well as their upper and their lower margin will be identified with each other. Such identifications will at least be done when they are Fourier transformed (using the DFT). They represent discrete periodic functions [18-20] and therewith build a bridge from distribution theory to linear algebra.

Zero-padding is moreover depicted in Figure 5. By adding zeros outside $\delta$ in "frequency domain" (left), we interpolate (inflate) 1 in "time domain" (right). More generally, zero-padding (discretizing) 
any scalar in one domain results in padding (periodizing) the scalar in the other domain. Vice versa, periodizing a scalar in one domain (replicating it), means discretizing the scalar in the other domain (inserting zeros). It reflects the already known duality between discretization and periodization [18].

\subsection{Pixels}

An important application of the two theorems above is to understand that $\delta$ is no mysterious object anymore that arises in "quantum mechanics" or in "distribution theory". It is simply what we know as number 1 or as "pixel" [31,32] in digital images (Figure 6).

$$
\left[\begin{array}{lllll}
0 & 0 & 0 & 0 & 0 \\
0 & 0 & 0 & 0 & 0 \\
0 & 0 & 1 & 0 & 0 \\
0 & 0 & 0 & 0 & 0 \\
0 & 0 & 0 & 0 & 0
\end{array}\right]
$$

Figure 6. A 2-D Dirac impulse. It is one of the $5 \times 5$ different basis images in Hilbert space $\mathbb{C}^{5 \times 5}$.

This has already been demonstrated in [32] where, on one hand, a digital image is written as finite double-sum of weigthed 2-D Dirac impulses. But using (4), this notation can be converted to a complex-valued matrix which is the superposition of complex-valued weigths multiplied by basis matrices that consistst of only one 1 at a certain position and zeros else (Dirac impulses) - in accordance with the results above.

\subsection{Resolution}

Quite the opposite of "pixel" is the concept of "resolution". While $[1,0, \ldots, 0]$ for example, defines a "pixel", its Fourier transform $[1,1, \ldots, 1]$ defines "resolution" (or "bandwidth") in the sense that 1 everywhere (globally) means "fully resolved" and the more ones are replaced by zeros in this domain the more resolution is lost. More precisely, $[1,1, \ldots, 1]$ actually means that the resolution cannot be any better because the Dirac delta $[1,0, \ldots, 0]$ cannot be any better focused and if the spectrum becomes rectangular-shaped $[0,1, \ldots, 1,0]$ then all $\delta$ (pixels) become sinc-shaped. This can be understood as the "spread-out" of one pixel's information to surrounding pixels. In that way, information is superimposed, hence, information (sharpness) is lost. Similarily, but the other way around, one may add zeros $[0,1, \ldots, 1,0]$ (see "zero-padding" above) at the margins of fully resolved data. This just interpolates data, will make them "smoother", but will not add any information, i.e., the image resolution remains unchanged. In engineering it is called "oversampling", "interpolation", "smoothing" or "zero-padding" [33], in generalized functions theory it is called "regularization" [34] and with respect to partial differential equations it is called "mollification" [35]. One may note that no information is lost in that way - despite the fact that the image appears unfocused now.

A standard case in radar imaging is it to have a rectangular-shaped "frequency spectrum". It is caused by a naturally arising finite bandwidth as it can be found in all real electronic systems. According to the classical sampling theorem, oversampling is required (see e.g. "oversampling makes it possible", [36], p.18; any factor "between 1.1 and 1.2" would be fine [37], p.131), i.e., the data spectrum is surrounded by zeros. The basis functions in digital (standard mode) synthetic aperture radar (SAR) images are, accordingly, sinc-functions [38] and in circular SAR modes they become Bessel-functions [31]. However, sinc-functions, are an expression of limited bandwidth. A usual technique therefore to measure "resolution" in radar images is to measure the widening of the sinc function mainlobe (at a level of factor $=0.707$ with respect to its top), e.g. Figure 2.3 in [37],[39]. Sinc-functions can also be thought of being regularized versions of Dirac deltas, see "regularization" 
in [19]. Vice versa, Dirac deltas can be thought of being the actual "kernel" information in sinc-functions. This can be seen by calculating the Fourier series of unity.

\subsection{Fourier Series of Unity}

The Fourier series of 1 can be calculated because 1 is a periodic function. Accordingly, (17) must be used rather than (15). We already know that $\mathcal{F} 1=\delta$, i.e., by calculating the Fourier series coefficients of the function that is constantly 1 , we actually calculate the individual samples of $\delta$. One may recall that if 1 is a periodic function then $\delta$ is a discrete function [18], i.e., $\delta$ will be defined at integers $m \in \mathbb{Z}$ rather than $t \in \mathbb{R}$.

So, our attention is especially directed to $m=0$ because it is commonly believed that $\delta$ would tend to infinity here - despite the fact that it is naturally been multiplied by 1 which should yield 1 at $m=0$ due to the sampling property of delta. However, using (17), we obtain

$$
\delta[m]=\frac{1}{T} \int_{-T / 2}^{+T / 2} e^{-2 \pi i \frac{m}{T} t} d t=\left.\frac{1}{T} \frac{e^{-2 \pi i \frac{m}{T} t}}{-2 \pi i \frac{m}{T}}\right|_{-T / 2} ^{+T / 2}=\frac{\left(e^{i \pi m}-e^{-i \pi m}\right)}{2 i \pi m}=\operatorname{sinc}[m]= \begin{cases}1, & m=0 \\ 0, & m \neq 0\end{cases}
$$

which tells us that $\delta$ is 1 at $m=0$ and zero else, independent of the actual choice of $T>0$. Thus, $\delta$ is a sinc function "in disguise". It coincides with the sinc function (at integers) but also with the Kronecker delta which is often considered (e.g. [40]) being the "discrete version" of $\delta$.

Let us recall at this point that Paul Dirac never claimed that $\delta$ must grow towards infinity. Instead, he claimed [29] that it is zero everywhere, except at the origin where its integral is unity - in accordance with the results above (Figure 3 ). The idea that $\delta$ is infinitely growing at the origin has later misleadingly been added by other authors.

\subsection{Delta applied to Delta}

In the above derivation, we actually asked for the "value" of $\delta$ at $t=0$ which corresponds to applying $\delta$ to $\delta$. We may summarize this in the following corollary.

Corollary 3. Let $\delta$ be Dirac's delta then

$$
\langle\delta, \delta\rangle=1
$$

in the sense of scalars (real or complex numbers).

Proof. We use $\delta \equiv 1$, hence $\langle\delta, \delta\rangle=\langle\delta, 1\rangle=1$ in the sense of scalars (real or complex numbers).

Alternatively, we may use the Fourier series argumentation

$$
\langle\delta, \delta\rangle=\langle\delta, \mathcal{F} 1\rangle=(\mathcal{F} 1)[0]=\delta[0]=\operatorname{sinc}[0]=1
$$

as derived above. Square brackets indicate the fact that a periodic function Fourier transformed yields a discrete function see (17), (18) or [20] for details.

\subsection{Dirac Delta Squared}

A Dirac delta according to the findings above is nothing else than the projection of a sinc-function (existing in a higher-dimensional space) to a lower dimensional space where only $\delta$ can be "seen". By projection we mean that a few of its dimensions (samples) were set to zero. It is, more precisely, the usual comprehension of "projection" in mathematics. Projections allow "seeing" higher-dimensional objects in lower-dimensional spaces. Standing behind a window for example, we only see two of the three dimensions of a 3-D object; one dimension is deleted in this case.

It is now not difficult anymore to assign a meaning to the Dirac delta squared $\delta^{2}$. It corresponds to looking at a squared sinc-function from a lower-dimensional space. One may note furthermore that 
this comprehension of a Dirac delta squared is in accordance with conventional Fourier series theory as seen above but also in accordance with modern distribution theory. The fact that modern distribution theory does not contradict conventional Fourier series theory is shown in [20]. In distribution theory,

$$
\delta^{2}=\delta
$$

means that asking two times for the particular sample of a function will always yield the same sample. Accordingly, powers of the Dirac delta correspond to asking several times for the same sample. This explanation

$$
\delta^{n}=\delta
$$

of powers of the Dirac delta is moreover consistent with most recent results in quantum mechanics [41], Definition 3.2.1.4, p.53. In other words, there is hope that this gap within distribution theory has indeed been closed now.

\subsection{Hilbert Spaces}

The "sinc" function has originally been introduced by Woodward because it "occurs so often in Fourier analysis and its applications that it does seem to merit some notation of its own" [42]. It was already clear at this time that it forms a Hilbert space basis [1,42]. Now, it remains to be seen what the results above actually mean. The fact that Kronecker's delta, the sinc function at integers and Dirac's delta actually coincide is at least encouraging. It confirms that spaces spanned by finite $[0, \ldots, 0,1,0, \ldots, 0]$ and by infinite vectors $[\ldots, 0,1,0, \ldots]$ create Hilbert spaces. But what does it mean for vectors of infinitely dence coordinates? At least in zero-dimensional spaces (e.g. in $\mathbb{R}^{0}$ ), one may write

$$
\|\delta\|=\sqrt{\langle\delta, \delta\rangle}=1
$$

which induces a norm such that $\mathbb{R}^{0}$ trivially becomes a Hilbert space.

\subsection{A Scalar's Integral}

Dirac demanded that his "new function" needs to be zero everywhere except at the origin where its integral is unity [29]. He could not imagine then that the scalar 1 itself would be his new function. Its "integral" is trivially unity as we see next. However, it is not the Lebesgue integral - this was the difficulty which had to be overcome via Schwartz' distribution theory $[12,13]$.

Corollary 4. Let 1 be unity (scalar). Then its integral is unity, symbolically

$$
\langle 1,1\rangle=1
$$

in the sense of scalars (real of complex numbers).

Proof. We use $\delta \equiv 1$, hence $\langle 1,1\rangle=\langle\delta, 1\rangle=1$ in the sense of scalars (real or complex numbers).

The symbol $\langle f, 1\rangle$ denotes the novel integral comprehension of a function $f$, as given in (13). One may briefly write $\langle f\rangle$ instead of $\langle f, 1\rangle$. The corollary complies with the idea that the Fourier transform actualy measures the entirety of all integrals of shifted versions of a function. In zero-dimensional spaces, however, there is no space for any shifts. Summarizing this corollary in one sentence, one may say that "every scalar arises as the measure of something" and trivially this "something" is not composed of anything else.

\subsection{Open Problems}

The above findings may help to resolve open problems because they there linked with each other, e.g. the multiplication among distributions [43-52], the division of distributions [53], the random 
square root of the Dirac delta [54] and the square of the Dirac delta [55] as already discussed above. A brief summary of the problem of the Dirac delta squared is given in [56].

\section{Conclusions}

We may summarize our results as follows. First, the Fourier transform of a scalar is the scalar itself and, consequently, the integral of any scalar is the scalar itself. Second, scalars are no functions. We may however think of scalars as trivial functions in the sense that they are both, discrete and periodic, simultaneously. They do also obey the Heisenberg uncertainty principle but trivially, they are best focused in both, time and frequency domain. Third, functions arise from scalars by either discretization, i.e., understanding them in an environment of zeros, or by periodization, i.e., repeating them as often as required, respectively. We moreover saw that unity can either be scalar, discrete or periodic in higher-dimensional spaces. However, this should not be accepted because unity should be unique. The following idea provides an alternative: We may identify any "inner" and "outer" unity with each other, i.e., the most "inner kernel" of any higher-dimensional space coincides with its "outer skin". If this can be done, a whole bunch of novel calculation rules will be needed.

Acknowledgments: The author would like to thank the German Aerospace Center (DLR) for generally providing the favorable underlying conditions and a great work atmosphere. The author would also like to thank his close colleagues at the German Aerospace Center, Microwaves and Radar Institute for their vigorous support.

Conflicts of Interest: The author declares no conflicts of interest.

\section{Distribution Theory}

One of the major principles in nature is the equivalence between smoothness and discreteness. It allows us for example to use the Discrete Fourier Transform (DFT) as if it were the integral Fourier transform [20]. This equivalence is moreover implicitly expressed in the overall setup of distribution theory. Distribution theory tells us a story about the equivalence between "regular" functions (functions in the ordinary functions sense which are smooth (infinitely differentiable) in the ordinary functions sense) and so-called "generalized" functions (which become smooth (infinitely differentiable) in the so-called "generalized" functions sense). They simply inherit their smoothness from "regular" functions and, vice versa, "regular" functions inherit their discreteness from "generalized" functions. The latter is expressed, for example, in the equivalence between Fourier series (smooth functions) and thier coefficients (discrete functions) or between polynomials (smooth functions) and their coefficients (discrete functions) or their zero crossings (discrete functions). One may also recall a considerable number of sampling theorems [57-66] or the wave-particle duality in physics [67,68], discussed in Appendix 9. An important application of "smoothness-discreteness" equivalences can be found in the field of "data compression" [69,70] or "pulse compression" [71] where smooth functions are reduced to discrete values and, vice versa, discrete values are used to restore smooth functions. The function that is constantly 1 (periodic function), for example, can be reduced to only one value 1 (discrete function) and, vice versa, one value 1 (discrete function) can be used to restore the whole function that is constantly 1 (periodic function).

The theory of generalized functions (distribution theory) had to be introduced to encounter an obvious contradiction triggered by the definition of sampling. One usually defines

$$
\int \delta(t) \varphi(t) d t:=\varphi(0)
$$

where the left-hand side is zero according to Lebesgue's integration theory and the right-hand side is non-zero. This definition obviously ignores the fact that the integral of functions which are non-zero at a single time instance, here $t=0$, must be zero in rigorous mathematical approaches. Ignoring this fact and continuing calculations on the basis of this definition would sooner or later lead to new contradictions. In particular, it would become impossible to distinguish "real" integrals which are zero from "artificial" integrals which are non-zero. Note that (11) "deletes" the original integral meaning on 
the left-hand side (which is zero) and assigns a new, artificial meaning to this integral (which is $\varphi(0)$ ). It follows that the original (Lebesgue) integral meaning is irretrievably lost in that way.

A trick to overcome this difficulty is to better introduce sampling as

$$
\langle\delta, \varphi\rangle:=\varphi(0)
$$

where we "apply" a Dirac $\delta$ (which is a discrete function) to a smooth function $\varphi$. Additionally, we more generally define

$$
\langle f, \varphi\rangle:=\int f(t) \varphi(t) d t
$$

as the "application" of generalized functions $f$ to smooth functions $\varphi$. The novelty is that arbitrarily defined new, more general "functions" $f$ can now be applied to other functions $\varphi$ without contradicting the conventional integral comprehension. One may notice, furthermore, that

$$
\langle f, 1\rangle=\int f(t) d t
$$

reduces to the usual Lebesgue integral. In particular, we have

$$
\langle\delta, 1\rangle=1
$$

as desired, i.e., the integral of $\delta$ is 1 - as demanded by Dirac $[29,40]$ although it is not the Lebesgue integral this time. However, now, we have no contradiction with respect to the Lebesgue integral. In other words, $\langle\cdot, \cdot\rangle$ denotes a novel integral comprehension. The idea can already be found in $[18,20,72]$. It includes both, Lebesgue's integral comprehension and Dirac's integral comprehension and is thereby implemented in the overall setup of "distribution theory". But as for ordinary integrals, we need to follow a few rules: Integrals should not become infinite. It is required, therefore, that $\langle f, \varphi\rangle<\infty$ whenever "functions" $f$ operate on functions $\varphi$. This can be secured by only allowing "certain" functions $f$ to operate on "certain" functions $\varphi$. The usual notation is to use $f \in X^{\prime}$ and to only apply them to $\varphi \in X$. One says that $X$ is the "test function space" of $X^{\prime}$.

In this study, we require two of these spaces, $\mathcal{E}^{\prime}$ and $\mathcal{S}^{\prime}$. A full range of distribution spaces can be found in $[13,18]$ for example. $\mathcal{S}^{\prime}$ is the space of tempered distributions. It greatly extends the space of square-integrable functions and it is a standard space in classical quantum mechanics and quantum field theory [41,73-80]. Tempered distributions operate on so-called Schwartz functions $\varphi \in \mathcal{S}$ which are smooth (infinitely differentiable) in the ordinary functions sense and do not grow faster than polynomials. The other space we need is $\mathcal{E}^{\prime}$. It is the space of compactly supported tempered distributions $\mathcal{E}^{\prime} \subset \mathcal{S}^{\prime}$. They operate on smooth (infinitely differentiable) functions $\varphi \in \mathcal{E}$. It is worth mentioning that in $\mathcal{E}$ we have no restrictions at all regarding the growth of test functions at infinity. We may waive such considerations here because $f \in \mathcal{E}^{\prime}$ is already compactly supported such that a growth of test functions at infinity would not be of any interest. In this study, we especially pay attention to $\mathcal{E}^{\prime}$ because the Dirac impulse $\delta \in \mathcal{E}^{\prime} \subset \mathcal{S}^{\prime}$ is a prominent member. It follows that everything we prove in $\mathcal{E}^{\prime}$ will automatically be true in $\mathcal{S}^{\prime}$, i.e., it is true "in the tempered distributions sense". This inclusion is especially important for applications in physics, in particular quantum mechanics, where $\mathcal{S}^{\prime}$ is a standard space.

The usual proof technique in distribution theory goes like this: In order to show that generalized functions $f \in X^{\prime}$ and $g \in X^{\prime}$ are equal in the sense of distributions, one must show that $\langle f, \varphi\rangle=\langle g, \varphi\rangle\langle\infty$ for any, arbitrarily chosen function $\varphi$ from their test function space $\mathrm{X}$. For any deeper introduction to distribution theory, the reader is invited to read brief introductions given in $[18,19]$. It remains to mention that in this study, in contrast to what is usually done, we implicitely use a space of constant test functions $\mathcal{E}_{0} \subset \mathcal{E}$ (see Theorem 1 and Theorem 2). We divide $\mathcal{E}$ into "constant test functions in $\mathcal{E}$ " on one hand and "non-constant test functions in $\mathcal{E}$ " on the other hand. This distinction has never been done in distribution theory - to the best of this authors knowledge. 


\section{Fourier Transforms and Fourier Series}

There are several ways of how to deal with a factor $2 \pi$ in Fourier transform and Fourier series definitions. The most symmetrical, however, is the so-called "unitary, ordinary frequency" Fourier transform [10], found e.g. in [8]. It leads to rules such as $\mathcal{F} \delta=1$ and $\mathcal{F} 1=\delta$. In that way, "time domain" and "frequency domain" become fully equivalent [20].

The Fourier Transform definition used in this and in our previous studies is

$$
\begin{array}{ll}
\hat{f}(\sigma):=\int_{-\infty}^{\infty} f(t) e^{-2 \pi i \sigma t} d t & \text { Analysis } \\
f(t)=\int_{-\infty}^{\infty} \hat{f}(\sigma) e^{2 \pi i t \sigma} d \sigma & \text { Synthesis }
\end{array}
$$

for non-periodic functions $f(t)$ and for periodic functions $f(t)$ with periods $T>0$ we use

$$
\begin{aligned}
& \hat{f}(m):=\frac{1}{T} \int_{0}^{T} \quad f(t) e^{-2 \pi i \frac{m}{T} t} d t \quad \text { Analysis } \\
& f(t)=\sum_{m=-\infty}^{\infty} \hat{f}(m) e^{2 \pi i \frac{t}{T} m} \quad \text { Synthesis }
\end{aligned}
$$

where (17) denotes the Fourier series coefficients (discrete function) and (18) denotes the Fourier series (periodic function). These definitions can moreover be used "in the distributional sense"

$$
\langle\mathcal{F} f, \varphi\rangle:=\langle f, \mathcal{F} \varphi\rangle
$$

i.e., by simply rolling them off to test functions $\varphi \in \mathcal{S}$. One may refer to [4,16,81].

The Fourier transform within $\mathcal{S}^{\prime}$ is - the same as within the space of square-integrable functions - an automorphism, i.e., the Fourier transform of a tempered distribution is again a tempered distribution. The space of tempered distributions moreover fully contains the space of square-integrable functions such that tempered distributions greatly extent the idea of square-integrable functions. The most prominent example of $\mathcal{F}$ in $\mathcal{S}^{\prime}$ is

$$
\mathcal{F} \delta=1
$$

because

$$
\langle\mathcal{F} \delta, \varphi\rangle=\left\langle\delta, \int_{-\infty}^{\infty} \varphi(t) e^{-2 \pi i \sigma t} d t\right\rangle=\int_{-\infty}^{\infty} \varphi(t) e^{0} d t=\langle 1, \varphi\rangle
$$

for arbitrary $\varphi \in \mathcal{S}$ and, vice versa,

$$
\mathcal{F} 1=\mathcal{F} \mathcal{F} \delta=\check{\delta}=\delta
$$

because $\delta$ is a symmetric distribution, i.e., $\check{\delta} \equiv \delta$. To see this, let $\check{\varphi}(t):=\varphi(-t)$ and $\langle\check{\delta}, \varphi\rangle:=\langle\delta, \breve{\varphi}\rangle$. It then follows that

$$
\langle\check{\delta}, \varphi\rangle=\langle\delta, \check{\varphi}\rangle=\check{\varphi}(0)=\varphi(0)=\langle\delta, \varphi\rangle
$$

for arbitrary $\varphi \in \mathcal{S},[81]$.

\section{Quantum Theory vs. Relativity Theory}

"One of the fundamental problems of modern physics is that two theories that have been proven correct, Quantum Theory and Relativity Theory, are basically not compatible. If both are correct representations of nature, how can they be so different and even contradictory? Relativity prescribes a spacetime continuum, whereas Quantum Theory prescribes a discrete world" [82,83].

Most recently (three years ago), it has been found that "Quantum physics just got less complicated: Wave-particle duality and quantum uncertainty are same thing" [67,84]. We have moreover seen that Heisenberg's uncertainty principle and Poisson's Summation Formula both represent dualities which 
are inverse to each other [18,19] and together they form a "smoothness-discreteness" duality, depicted in Figure 2, which appears to be one of the major principles in nature. We may therefore think of the wave-particle duality as one instance of a "smoothness-discreteness" duality where waves are smooth and particles are discrete.

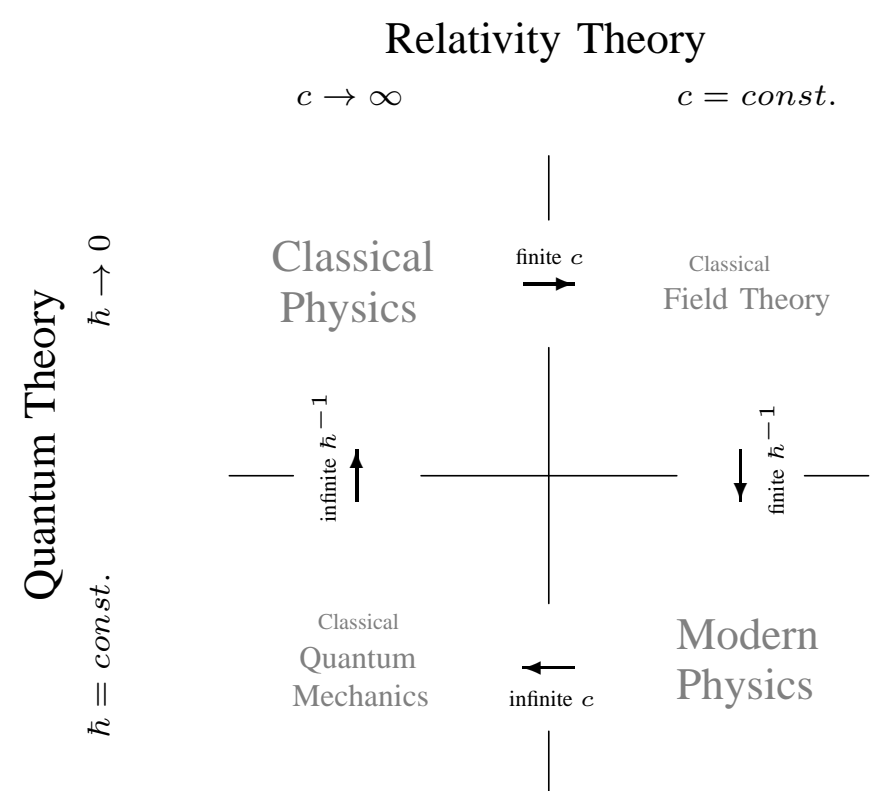

Figure 7. Quantum Theory vs. Relativity Theory.

This principle not only connects the microscopic world (Quantum Theory) to the macroscopic world (Relativity Theory) as depicted in Figure 7, it also emerges within these worlds. One may recall for example that the behavior of particles is described via Hermite functions [75], i.e., via infinitely smooth functions in $\mathcal{S}$, test functions of tempered distributions $\mathcal{S}^{\prime}$ (rough functions). This "smoothness-discreteness" duality in nature is moreover a particular case of Born's principle of reciprocity [21-26] because smoothness is the reciprocal (Fourier transform) of discreteness, and, vice versa, discreteness is the reciprocal (Fourier transform) of smoothness [18-20]. It is moreover consistent to Scaruffi's idea of a (smooth) ocean (Relativity Theory) and its (rough) ripples (Quantum Theory), [82].

The fact that $\hbar^{-1}$ and $c^{+1}$ are finite entities in our universe - one may say $\hbar$ and $c$ depict "lower" and "upper" bounds of the universe - suggests that we are living in a "fully discrete world". One may compare Figure 2 to Figure 7 (see [74], Figure 1.1). This conclusion can be drawn as we have in mind that "finiteness" and "periodicity" (= "reciprocal discreteness") correspond to each other [20] and, vice versa, "reciprocal finiteness" and "discreteness" (= "reciprocal periodicity") correspond to each other. It is thus expected that the universe will appear discrete and cyclic to us because it is of finite extent in both, microscopic and macroscopic dimensions.

Very interesting, furthermore, is to think of "time" as an entity that runs from discreteness to smoothness or, alternatively, from smoothness to discreteness. Whether the actual direction is "forward" or "backward" depends on the point of view. The first idea corresponds to Linde's chaotic inflation theory [85]. The universe, very chaotic and very rough in the beginning, behaves "just like a deflated balloon" and "appears to be a contorted shape", gets smoother and smoother and finally becomes "a perfect sphere" the more it is "inflated" [82]. In contrast to that, the opposite idea applies to human beings: They have smooth skin "in the beginning" and get an increasingly wrinkled skin the older they get. 


\section{References}

1. Woodward, P.M. Probability and Information Theory, with Applications to Radar; Pergamon Press, 1953.

2. Bracewell, R.N. Fourier Transform and its Applications; McGraw-Hill Education, 1986.

3. Benedetto, J.J. Harmonic Analysis and Applications; Vol. 23, CRC Press, 1996.

4. Gasquet, C.; Witomski, P. Fourier Analysis and Applications: Filtering, Numerical Computation, Wavelets; Vol. 30, Springer Science \& Business Media, 1999.

5. Gröchenig, K. Foundations of Time-Frequency Analysis; Birkhäuser, 2001.

6. Brandwood, D. Fourier Transforms in Radar and Signal Processing; Artech House, 2003.

7. Strichartz, R.S. A Guide to Distribution Theorie and Fourier Transforms; World Scientific, 2003.

8. Kammler, D.W. A First Course in Fourier Analysis; Cambridge University Press, 2007.

9. Fischer, J. Calculation Rules for Discrete Functions, Periodic Functions and Discrete Periodic Functions. ResearchGate, https://doi.org/10.13140/rg.2.2.21779.07201 2017.

10. Wikipedia, Fourier Transform. https://en.wikipedia.org/wiki/Fourier\%20transform 2017.

11. Wikipedia, Dirac Delta Function. https://en.wikipedia.org/wiki/Dirac_delta_function.

12. Schwartz, L. Théorie des Distributions, Tome I; Hermann Paris, France, 1950.

13. Schwartz, L. Théorie des Distributions, Tome II; Hermann Paris, France, 1951.

14. Lighthill, M.J. An Introduction to Fourier Analysis and Generalised Functions; Cambridge University Press, 1958.

15. Jones, D. The Theory of Generalized Functions; Cambridge University Press, 1982.

16. Walter, W. Einführung in die Theorie der Distributionen; BI-Wissenschaftsverlag, Bibliographisches Institut \& FA Brockhaus, 1994.

17. Vladimirov, V.S. Methods of the Theory of Generalized Functions; CRC Press, 2002.

18. Fischer, J.V. On the Duality of Discrete and Periodic Functions. Mathematics 2015, 3, 299-318.

19. Fischer, J.V. On the Duality of Regular and Local Functions. Mathematics, 5, 41.

20. Fischer, J.V. There is only one Fourier Transform. Preprints 2017, 2017120173, https://doi.org/10.20944/preprints201712.0173.v1.

21. Born, M.; Infeld, L. Foundations of the New Field Theory. Proceedings of the Royal Society of London. Series A, Containing Papers of a Mathematical and Physical Character 1934, 144, 425-451.

22. Born, M. A Suggestion for Unifying Quantum Theory and Relativity. Proceedings of the Royal Society of London A: Mathematical, Physical and Engineering Sciences. The Royal Society, 1938, Vol. 165, pp. 291-303.

23. Born, M. Relativity and Quantum Theory. Nature 1938, 141, 327-328.

24. Born, M. Elementary Particles and the Principle of Reciprocity. Nature 1949, 163, 207-208.

25. Green, H. Quantized Field Theories and the Principle of Reciprocity. Nature 1949, 163, 208-9.

26. Mignemi, S.; Štrajn, R. Quantum Mechanics on a Curved Snyder Space. Advances in High Energy Physics 2016.

27. Wikipedia, Parseval's theorem. https://en.wikipedia.org/wiki/Parseval\%27s_theorem 2017.

28. Born, M. Reciprocity Theory of Elementary Particles. Reviews of Modern Physics 1949, 21, 463.

29. Dirac, P.A.M. The Principles of Quantum Mechanics, Forth Edition; Oxford University Press, 1958.

30. Rosinger, E.E. What Scalars Should We Use? arXiv preprint math/0505336 2005.

31. Ponce, O.; Prats-Iraola, P.; Pinheiro, M.; Rodriguez-Cassola, M.; Scheiber, R.; Reigber, A.; Moreira, A. Fully Polarimetric High-resolution 3-D Imaging with Circular SAR at L-Band. IEEE Transactions on Geoscience and Remote Sensing 2014, 52, 3074-3090.

32. Fischer, J.; Molkenthin, T.; Chandra, M. SAR Image Formation as Wavelet Transform. Proceedings of EUSAR 2006.

33. Smith, J.O.; Serra, X. PARSHL: An Analysis/Synthesis Program for Non-harmonic Sounds based on a Sinusoidal Representation; CCRMA, Department of Music, Stanford University, 1987.

34. Hörmander, L. The Analysis of Linear Partial Differential Operators I; Die Grundlehren der mathematischen Wissenschaften, Springer, 1983.

35. Schechter, M. Modern Methods in Partial Differential Equations, An Introduction, 1977.

36. Daubechies, I. The Wavelet Transform, Time-Frequency Localization and Signal Analysis. Information Theory, IEEE Transactions on 1990, 36, 961-1005. 
37. Cumming, I.G.; Wong, F.H. Digital Processing of Synthetic Aperture Radar Data. Artech house 2005, 1, 3.

38. Fischer, J.; Pupeza, I.; Scheiber, R. Sidelobe Suppression using the SVA Method for SAR Images and Sounding Radars 2006.

39. Klausing, H.; Holpp, W. Radar mit realer und synthetischer Apertur: Konzeption und Realisierung; Walter de Gruyter, 1999.

40. Debnath, L. Developments of the Theory of Generalized Functions or Distributions - A Vision of Paul Dirac. Analysis - International Mathematical Journal of Analysis and its Applications 2013, 33, 57-99.

41. Maroun, M.A. Generalized Quantum Theory and Mathematical Foundations of Quantum Field Theory. PhD thesis, 2013.

42. Woodward, P.M.; Davies, I.L. Information Theory and Inverse Probability in Telecommunication. Proceedings of the IEE-Part III: Radio and Communication Engineering 1952, 99, 37-44.

43. Fisher, B. Some Results on the Product of Distributions. Mathematical Proceedings of the Cambridge Philosophical Society. Cambridge University Press, 1973, Vol. 73, pp. 317-325.

44. Oberguggenberger, M.B. Multiplication of Distributions and Applications to Partial Differential Equations; Vol. 259, Longman Scientific \& Technical, Harlow, Essex, U.K., 1992.

45. Colombeau, J. A Multiplication of Distributions. Journal of Mathematical Analysis and Applications 1983, 94, 96-115.

46. Colombeau, J.F. Multiplication of Distributions: A Tool in Mathematics, Numerical Engineering and Theoretical Physics; Springer, Berlin, Heidelberg, 1992.

47. Colombeau, J.; Meril, A. Generalized Functions and Multiplication of Distributions on $C^{\infty}$ manifolds. Journal of Mathematical Analysis and Applications 1994, 186, 357-364.

48. Gsponer, A. The Sequence of Ideas in a Re-discovery of the Colombeau Algebras. arXiv preprint arXiv:0807.0529 2008.

49. Gsponer, A. A Concise Introduction to Colombeau Generalized Functions and their Applications in Classical Electrodynamics. European Journal of Physics 2008, 30, 109.

50. Gsponer, A. The Classical Point Electron in Colombeau's Theory of Nonlinear Generalized Functions. Journal of Mathematical Physics 2008, 49, 102901.

51. Gsponer, A. The Self-interaction Force on an Arbitrarily Moving Point-charge and its Energy-momentum Radiation Rate: A Mathematically Rigorous Derivation of the Lorentz-Dirac Equation of Motion. arXiv preprint arXiv:0812.3493 2008.

52. Gsponer, A. First-order Quantum Perturbation Theory and Colombeau Generalized Functions. arXiv preprint arXiv:0809.2576 2008.

53. Rosinger, E. Division of Distributions. Pacific Journal of Mathematics 1976, 66, 257-263.

54. Si, S. T-Transform and S-Transform on the Space of Hida Distributions 2009.

55. Mikusiński, J. On the Square of the Dirac Delta-Distribution. Bull. Polish Acad. Sci. Math. 1966, pp. 511-513.

56. Trenn, S. Regularity of Distributional Differential Algebraic Equations. Mathematics of Control, Signals, and Systems (MCSS) 2009, 21, 229-264.

57. Holschneider, M. An Analysis Tool; Oxford Mathematical Monographs, Clarendon Press, 1995.

58. Linden, D. A Discussion of Sampling Theorems. Proceedings of the IRE 1959, 47, 1219-1226.

59. Campbell, L. A Comparison of the Sampling Theorems of Kramer and Whittaker. Journal of the Society for Industrial and Applied Mathematics 1964, 12, 117-130.

60. Stark, H. Sampling Theorems in Polar Coordinates. JOSA 1979, 69, 1519-1525.

61. Nashed, M.Z.; Walter, G.G. General Sampling Theorems for Functions in Reproducing Kernel Hilbert Spaces. Mathematics of Control, Signals and Systems 1991, 4, 363-390.

62. Feichtinger, H.G.; Gröchenig, K. Irregular Sampling Theorems and Series Expansions of Band-limited Functions. Journal of mathematical analysis and applications 1992, 167, 530-556.

63. Janssen, A.J. The Zak Transform and Sampling Theorems for Wavelet Subspaces. IEEE Transactions on Signal Processing 1993, 41, 3360-3364.

64. Djbkovic, I.; Vaidyanathan, P. Generalized Sampling Theorems in Multiresolution Subspaces. IEEE Transactions on Signal Processing 1997, 45, 583-599.

65. Chen, W.; Itoh, S.; Shiki, J. Irregular Sampling Theorems for Wavelet Subspaces. IEEE Transactions on Information Theory 1998, 44, 1131-1142. 
66. Blumensath, T.; Davies, M.E. Sampling Theorems for Signals from the Union of Finite-dimensional Linear Subspaces. IEEE Transactions on Information Theory 2009, 55, 1872-1882.

67. Coles, P.J.; Kaniewski, J.; Wehner, S. Equivalence of Wave-Particle Duality to Entropic Uncertainty. Nature Communications 2014, 5, 5814.

68. Lake, M. Which Quantum Theory Must be Reconciled with Gravity? (And What Does it Mean for Black Holes?). Universe 2016.

69. Fischer, J.; Benz, U.; Moreira, A. Efficient SAR Raw Data Compression in Frequency Domain. Proceedings of the IEEE International Geoscience and Remote Sensing Symposium (IGARSS), 1999, Vol. 4, pp. 2261-2263.

70. Benz, U.; Fischer, J.; Moreira, A. Compression and Decompression Method for Complex Image Data from a Synthetic Aperture Radar, Patent, 2002. EP000000959369B1.

71. Fischer, J.; Molkenthin, T.; Chandra, M. A Direct Comparison of SAR Processing as Non-Orthogonal Transform to both Fourier and Wavelet Transform. Proceedings of Wave Propagation in Communication, Microwaves Systems and Navigation (WFMN) 2007, pp. 91-96.

72. Horváth, J. Topological Vector Spaces and Distributions; Addison-Wesley Publishing Company, 1966.

73. Messiah, A. Quantum Mechanics, Two Volumes in One; Dover, Mineola, New York, 1999.

74. Glimm, J.; Jaffe, A. Quantum Physics: A Functional Integral Point of View; Springer Science \& Business Media, 2012.

75. Simon, B. Distributions and their Hermite Expansions. Journal of Mathematical Physics 1971, 12, $140-148$.

76. Maillard, J. On the Twisted Convolution Product and the Weyl Transformation of Tempered Distributions. Journal of geometry and physics 1986, 3, 231-261.

77. Gracia-Bondía, J.M.; Várilly, J.C. Algebras of Distributions Suitable for Phase-space Quantum Mechanics. I. Journal of Mathematical Physics 1988, 29, 869-879.

78. Várilly, J.C.; Gracia-Bondía, J.M. Algebras of Distributions Suitable for Phase-space Quantum Mechanics. II. Topologies on the Moyal algebra. Journal of Mathematical Physics 1988, 29, 880-887.

79. Dias, N.C.; de Gosson, M.; Luef, F.; Prata, J.N. Quantum Mechanics in Phase Space: the Schrödinger and the Moyal Representations. Journal of Pseudo-Differential Operators and Applications 2012, pp. 1-32.

80. Becnel, J.; Sengupta, A. The Schwartz Space: Tools for Quantum Mechanics and Infinite Dimensional Analysis. Mathematics 2015, 3, 527-562.

81. Fischer, J. Anwendung der Theorie der Distributionen auf ein Problem in der Signalverarbeitung. Diploma thesis, Ludwig-Maximilians-Universität (LMU) München, doi 10.13140/RG.2.2.25635.86560, 1997.

82. Scaruffi, P. On the Relationship between Quantum Theory and Relativity Theory, 2004, https://www.scaruffi.com/science/physics.html.

83. Yilmaz, H. Relativity and Quantum Mechanics. International Journal of Theoretical Physics 1982, 21, 871-902.

84. ScienceDaily. Quantum Physics Just Got Less Complicated: Wave-Particle Duality and Quantum Uncertainty are Same Thing, 2004, https:/ / www.sciencedaily.com/releases/2014/12/141219085153.htm.

85. Linde, A.D. Chaotic Inflation. Physics Letters B 1983, 129, 177-181. 